\title{
NONLINEAR LIE DERIVATIONS OF INCIDENCE ALGEBRAS
}

\author{
YUPING YANG
}

Abstract. Let $(X, \leqslant)$ be a locally finite preordered set and $R$ a 2 -torsion free commutative ring with unity, $I(X, R)$ the incidence algebra of $X$ over $R$. In this paper, we give an explicit description of the structure of nonlinear Lie derivations of $I(X, R)$. We prove that every nonlinear Lie derivation of $I(X, R)$ is a sum of an inner derivation, a transitive induced derivation and an additive induced Lie derivation.

Mathematics subject classification (2010): Primary 16S40, 16W25; Secondary 16G20, 06A11, 47L35. Keywords and phrases: Derivation, nonlinear Lie derivation, incidence algebra.

\section{REFERENCES}

[1] K. Baclaws Ki, Automorphisms and derivations of incidence algebras, Proc. Amer. Math. Soc. 36 (1972), 351-356.

[2] Z.-F. BAI AND S.-P. DU, The structure of nonlinear Lie derivation on von Neumann algebras, Linear Algebra Appl. 436 (2012), 2701-2708.

[3] D. BenkoviČ AND D. EREmita, Multiplicative Lie $n$-derivations of triangular rings, Linear Algebra Appl. 436 (2012), 4223-4240.

[4] M. BREŠAR, Commuting traces of biadditive mappings, commutativity-preserving mappings and Lie mappings, Trans. Amer. Math. Soc. 335 (1993), 525-546.

[5] M. Brešar, M. A. Chebotar And W. S. Martindale III, Functional Identities, Birkhäuser Verlag, 2007.

[6] R. Brusamarello, É. Z. Fornaroli and E. A. SAntulo, Anti-automorphisms and involutions on (finitary) incidence algebras, Linear Multilinear Algebra 60 (2012), 181-188.

[7] R. BRUs amarello AND D. Lewis, Automorphisms and involutions on incidence algebras, Linear Multilinear Algebra 59 (2011), 1247-1267.

[8] L. Chen AND J.-H. ZhANG, Nonlinear Lie derivations on upper triangular matrices, Linear Multilinear Algebra 56 (2008), 725-730.

[9] B. DhaRA AND S. ALI, On multiplicative (generalized)-derivations in prime and semiprime rings, Aequ. Math. 86 (2013), 65-79.

[10] A. FOŠNER, commutativity preserving maps on $\mathrm{M}_{n}(\mathbb{R})$, Glasnik. Mat. 44 (2009), 127-140.

[11] A. Fošner, F. WEI AND Z.-K. XIAO, Nonlinear Lie-type derivations of von Neumann algebras and related topics, Colloq. Math. 132 (2013), 53-71.

[12] H. Goldmann, AND P. ŠEmRL, Multiplicative derivations on $C(X)$, Monatsh. Math. 121 (1996), 189-197.

[13] I. N. HersteIn, Lie and Jordan structures in simple, associative rings, Bull. Amer. Math. Soc. 67 (1961), 517-531.

[14] P.-S. Ji, R.-R. LiU AND Y.-Z. ZhaO, Nonlinear Lie triple derivations of triangular algebras, Linear Multilinear Algebra 60 (2012), 1155-1164.

[15] H.-Y. JiA AND Z.-K. XIAO, Commuting maps on certain incidence algebras, Bull. Iran. Math. Soc. (2019), https://doi.org/10.1007/s41980-019-00289-1.

[16] I. KAYGORODOV, M. KHRYPCHENKO, AND F. WEI, Higher derivations of finitary incidence rings, Algebr. Represent. Theory (2018), https ://doi .org/10.1007/s10468-018-9822-4.

[17] M. KhrypChEnKo, Jordan derivations of finitary incidence rings, Linear Multilinear Algebra 64 (2016), 2104-2118. 
[18] M. Koppinen, Automorphisms and higher derivations of incidence algebras, J. Algebra, 174 (1995), 698-723.

[19] W. S. Martindale III, When are multiplicative mappings additive?, Proc. Amer. Math. Soc. 21 (1969), 695-698.

[20] F. Y. LU, Jordan derivable maps of prime rings, Comm. Algebra. 38 (2010), 4430-4440.

[21] F. Y. Lu AND B. H. LiU, Lie derivable maps on B(X), J. Math. Anal. Appl. 372 (2010), 369-376.

[22] L. MOLNÁR AND P. ŠEMRL, Elementary operators on standard operator algebras, Linear Multilinear Algebra, 50 (2002), 315-319.

[23] A. NowICKI, Derivations of special subrings of matrix rings and regular graphs, Tsukuba. J. Math. 7 (1983), 281-297.

[24] P. ŠEMrL, Nonlinear commutativity preserving maps, Acta Sci. Math. (Szeged) 71 (2005), 781-819.

[25] E. SPIEGEL, On the automorphisms of incidence algebras, J. Algebra, 239 (2001), 615-623.

[26] E. Spiegel And C. O'Donnell, Incidence algebras, Monographs and Textbooks in Pure and Applied Mathematics, vol. 206, Marcel Dekker, New York, 1997.

[27] R. STANLEY, Structure of incidence algebras and their automorphism groups, Bull. Amer. Math. Soc. 76 (1970), 1236-1239.

[28] Y. WANG AND Y. WANG, Multiplicative Lie n-derivations of generalized matrix algebras, Linear Algebra Appl. 438 (2013), 2599-2616.

[29] D.-N. WANG AND Z.-K. XIAO, Lie triple derivations of incidence algebras, Comm. Algebra, 47 (2019), 1841-1852.

[30] Z.-K. XIAO, Jordan derivations of incidence algebras, Rocky Mountain J. Math. 45 (2015), 13571368.

[31] Z.-K. XIAO AND F. WEI, Nonlinear Lie-type derivations on full matrix algebras, Monatsh. Math. 170 (2013), 77-88.

[32] Y.-P. YANG, Nonlinear Lie derivations of incidence algebras of finite rank, Linear Multilinear Algebra, (2019) https://doi .org/10.1080/03081087.2019.1635979.

[33] W.-Y. YU AND J.-H. ZhANG, Nonlinear Lie derivations of triangular algebras, Linear Algebra Appl. 432 (2010), 2953-2960.

[34] X. Zhang And M. Khrypchenko, Lie derivations of incidence algebras, Linear Algebra Appl. 513 (2017), 69-83. 